\title{
A single sub-anaesthetic dose of propofol to reduce patient recall of peribulbar block
}

\author{
G Hocking, HGR Balmer
}

\begin{abstract}
We audited a total of 1233 patients scheduled for elective cataract extraction or trabeculectomy using peri-bulbar anaesthesia. A bolus of propofol provided sedation to cover insertion of the block. During an initial two-year period we collected data on the amount of propofol used to sedate 481 patients. Multiple linear regression analysis was then used to obtain an equation to link the dose used to age and weight in those who were adequately sedated. The dose of propofol in milligrams was calculated as $56+0.25 \mathrm{x}$ weight (kg) - $0.53 \mathrm{x}$ age (yrs). We subsequently assessed the effectiveness of this formula at abolishing recall of the injections whilst not compromising the airway on a further 752 patients. This simple regime was effective at abolishing recall of the block in $78.6 \%$ of the patients studied and avoids the cost and logistic implications of more complicated drug delivery systems. Use of the formula does not significantly alter the incidence of recall compared to sedation provided by an experienced ophthalmic anaesthetist. We hope it will provide a guide for more junior anaesthetists to obtain the satisfactory sedation level that comes with experience.
\end{abstract}

\section{Keywords}

anaesthetics i.v., propofol; anaesthetics local; surgery ophthalmological

\section{Introduction}

Ophthalmic surgery using peri-bulbar local anaesthesia is a well-established practice.

G Hocking DMCC DA FRCA RAF.

Specialist Registrar in

Anaesthetics.

Department of

Anaesthesia, MDHU

Frimley Park Hospital,

Frimley, Camberley,

Surrey, GU16 5UJ

E-mail:

drghocking@hotmail.com

H G R Balmer FRCA

Consultant Anaesthetist.

Department of

Anaesthesia, Derriford

Hospital,

PLYMOUTH, Devon.

PL6 8DH

Many methods of sedation have been tried to reduce patient anxiety and improve acceptance of the technique, however these often increase the risks of oxygen desaturation (1), sudden movement with disorientation on wakening and ocular field movement during surgery (2). Propofol has been shown to reduce intraocular pressure (3) and reduce patient recall following peribulbar injections (4). Since the duration of action is shorter than many other sedative agents, it is also likely that there would be a faster return to "street fitness". We audited our routine practice to find the optimal single bolus dose of propofol that prevented recall without causing excessive sedation then assessed it prospectively.

\section{Methods \\ Stage 1: Analysis Stage (Group A)}

We collected data on 481 patients scheduled for elective cataract extraction or trabeculectomy using peri-bulbar anaesthesia and propofol sedation. All patients gave fully informed written consent for the surgical procedure and sedation. Monitoring consisted of continuous pulse oximetry with non-invasive blood pressure measurements at the beginning and at the end of the procedure in accordance with routine practice. Intravenous access was obtained and $0.5 \%$ proxymetacaine eye drops instilled. The patient was then given a bolus injection of propofol at a dose based on the clinical judgement of an experienced ophthalmic anaesthetist. After 2-3 minutes a standard transcutaneous peribulbar block was performed. This was administered as a 3-4ml infra-temporal injection followed by a $2-3 \mathrm{ml}$ supero-medial injection using a local anaesthetic mixture with final concentrations of lignocaine $2 \%$, bupivacaine $0.25 \%$, adrenaline $1: 200,000$ and hyaluronidase $15 \mathrm{i} . \mathrm{u} . / \mathrm{ml}$. The blocks took 60-90 seconds to complete. Oxygen saturation prior to sedation, the lowest level reached during the block, age and weight were recorded. The block was tested for adequacy after 10 minutes. At this time the patient was asked "Do you remember me putting a needle into you?" Those who did were then asked, "Where did I put it?" Sedation was graded either: a. Inadequate (patient recall); b. Good (no recall); c. Excessive (no recall but some form of airway support required).
Stage 2: Prospective Stage (Group P)

We collected data on a further 752 patients scheduled for elective trabeculectomy or cataract extraction under peribulbar anaesthesia. The propofol dose administered for sedation was calculated on the basis of the patients' age and weight according to the formula obtained in stage 1. The remainder of the methodology was as described above.

\section{Statistical Analysis}

Statistical analysis was performed using Microsoft Excel 5.0 multiple linear regression analysis software running on a personal computer. Using the data from the group of patients with no recall, a formula 
for the optimal propofol dose was obtained based on age and weight.

\section{Results}

Patient demographics are shown in table 1. During the analysis stage $21.3 \%(n=102)$ of patients had recall of the block, $78.1 \%$ $(n=376)$ no recall and $0.6 \%(n=3)$ were excessively sedated. Multiple regression analysis of the successful cases from stage 1 provided the equation: propofol dose $(\mathrm{mg})=56 \mathrm{mg}+($ weight $\mathrm{x} 0.25)-($ age $\mathrm{x}$ 0.53) where weight is expressed in kilograms and age in years. Ninety-five percent confidence intervals for the coefficients and constant were: weight 0.20 to 0.30 , age -0.45 to 0.60 and intercept 48 to 64 . Analysis of the patient group with recall produced similar results. During the prospective stage $21.1 \% \quad(n=159)$ had recall, $78.6 \%(n=591)$ no recall and $0.3 \%$ $(n=2)$ were excessively sedated. Grimacing, movement or vocalisation during the block did not correlate with patient recall. The commonest reply to the question regarding "where did I put the needle?" was "In my hand", recalling venous cannulation rather than the peri-bulbar block.

Airway support (chin lift) was required in $0.6 \%$ patients from group $A(n=3)$ and $0.3 \%$ patients from group $P(n=2)$. No other airway intervention was needed in 4 of these 5 patients. Oxygen was immediately available but only needed in one patient due to hypoxia. No patients with inadequate sedation had oxygen saturation lower than $90 \%$ at any time during the block. Transient oxygen desaturation ( $<90 \%$ for less than 60 secs) in the group with adequate sedation was recorded in $2.9 \%$ of group A patients $(n=14)$ and $2.3 \%$ group $P$ patients $(n=17)$. Only $1.2 \%$ of patients $(n=15)$ in total had a reduction in oxygen saturation of greater than $8 \%$ from pre-sedation values; one third of these occurred in the group with presedation values already below $92 \%$. Of the 1233 patients studied, 9\% $(n=111)$ had greater than $4 \%$ reduction in oxygen saturation following the propofol bolus. The inadequately sedated group contained both a greater percentage of males, and a greater proportion of the younger patients in both the analysis and validation groups. There were no differences between the two groups regarding the timings of eye drops, sedation or needle insertion and removal.

\section{Discussion}

When explaining the technique of ophthalmic surgery under local anaesthesia to patients they are often anxious about the thought of needle infiltration near the eyes, and it is therefore common practice to use some form of sedation. Midazolam (4), ketamine (5), alfentanil $(6,7)$, methohexital $(4,6,8)$ and propofol $(4,9)$ have all been used for this purpose with little to choose between them in terms of patient recall, sedation and postoperative amnesia (4). With the exception of propofol, they can all produce prolonged sedation, which can lead to patients falling asleep during surgery with sudden movement upon wakening. Propofol has a shorter sedative end-point and avoids intra-operative somnolence. There is also some evidence that there is a reduced incidence of postoperative nausea and vomiting (10), lower intraocular pressure (3) and earlier readiness for discharge (4). Recently the use of patient controlled sedation with propofol $(11,12)$ or midazolam (12) during surgery has been shown to improve patient satisfaction with no difference in the incidence of perioperative complications. There is evidence that patients find simple premedication with temazepam and metoclopramide as acceptable for anxiolysis as intravenous sedation with either propofol or midazolam (2) but this does not abolish recall. Studies using patient controlled sedation with propofol show that $50 \%$ of the total administered dose is given prior to the peribulbar block being performed and most of the remainder at the beginning of surgery (12). We suggest that a single dose of propofol prior to administering the block can achieve the goal of improving patient acceptance through reduced recall. This is particularly important when patients are likely to need to return for surgery on the other eye. The incidence of non-recall is acceptable compared to other studies although in retrospect we could have also assessed patient satisfaction and graded the degree of recall, since not all recall may have been unpleasant. Whilst using the formula has not significantly altered the incidence of recall, its use by more inexperienced anaesthetists may allow them to obtain the satisfactory levels of sedation which the experienced anaesthetist can produce through good judgement. Our technique also avoids the problems associated with longer-acting sedatives and the cost implications of patient-controlled infusion devices. Supplemental oxygen should be given through nasal cannulae to those patients with pre-sedation oxygen saturation of $94 \%$ or less.

More males seem to be recalling the block suggesting that they are being undersedated. We have analysed the data from the prospective group and repeated the multiple regression analysis on the adequately sedated patients after subdividing them by sex. Two different equations are produced which give the males a different dose of propofol:

Male dose $(\mathrm{mg})=70+0.21 x$ weight $-0.65 x$ age Female dose $(m g)=71+0.29 x$ weight $-0.74 x$ age

However, we have not formally assessed these new formulae in clinical practice. 
Indeed, using these formulae on computer generated male patients, they do not always receive higher doses than females as would be expected from our prospective data. There is also the potential for a greater incidence of excessive sedation and airway compromise from increased doses of propofol with perhaps only a small decrease in patient recall. Since some anaesthetists avoid sedation altogether, for the reasons discussed earlier, we feel that our non-recall rate of almost $80 \%$ is acceptable and introduction of sex related, more complicated formulae would only detract from this simple technique.

In summary, we have devised a simple formula for a single bolus propofol technique to reduce recall during insertion of a peribulbar block. For clinical use this could be simplified to $56 \mathrm{mg}+(0.25 x$ weight $)-$ $(0.5 x$ age $)$. We have prospectively assessed its effectiveness and safety on a total of 752 patients with minimal morbidity.

Table 1. Demographic data

\begin{tabular}{lcccc}
\hline & \multicolumn{2}{c}{$\begin{array}{c}\text { Analysis Group }(\boldsymbol{A}) \\
(\mathrm{n}=481)\end{array}$} & \multicolumn{2}{c}{$\begin{array}{c}\text { Prospective group }(\boldsymbol{P}) \\
(\mathrm{n}=752)\end{array}$} \\
\hline $\begin{array}{l}\text { Sedation } \\
(\%)\end{array}$ & Inadequate & Good & Inadequate & Good \\
\hline Male/Female $(\%)$ & 21.3 & 78.1 & 21.1 & 78.7 \\
Weight $(\mathrm{kg})^{\star}$ & $45: 55$ & $34: 66$ & $48: 52$ & $34: 66$ \\
Age $(\mathrm{yrs})^{\star}$ & $70(15)$ & $68(15)$ & $72(13.5)$ & $68(15)$ \\
\hline
\end{tabular}

${ }^{\star}$ Results expressed as mean (standard deviation)

\section{References}

1. Wong DH, Merick PM. Intravenous sedation prior to peribulbar anaesthesia for cataract surgery in elderly patients. Can F. Anaesth 1996; 43(11): $1115-20$

2. Salmon JF, Mets B, James MF, Murry AD. Intravenous sedation for ocular surgery under local anaesthesia. Br. F. Ophthalmol 1992; 76(10): 598601

3. Lauretti GR, Lauretti CR, Lauretti-Filho A. Propofol decreases ocular pressure in outpatients undergoing trabeculectomy. F. Clin. Anesth 1997; 9(4): 289-92

4. Ferrari LR, Donlon JV. A comparison of propofol, midazolam, and methohexital for sedation during retrobulbar and peribulbar block. F. Clin. Anesth. 1992; 4(2): 93-6

5. Rosenberg MK, Raymond C, Bridge PD. Comparison of midazolam/ketamine with methohexital for sedation during peribulbar block. Anesth. Analg. 1995; 81(1): 173-4

6. Yee JB, Schafer PG, Crandall AS, Pace NL. Comparison of methohexital and alfentanil on movement during placement of retrobulbar nerve block. Anesth. Analg 1994; 79: 320-3

7. Stead SW, Northfield KM. Effects of alfentanil analgesia for ophthalmic nerve blocks (abstract). Anesthesiology 1990; 73: A769

8. Gilbert J, Holt JE, Johnston J, Sabo BA and Weaver $\mathrm{J}$ S. Intravenous sedation for cataract surgery. Anaesthesia 1987; 42: 1063-9

9. Beatie CD, Stead SW. Effects of propofol sedation for ophthalmic nerve blocks (abstract). Anesthesiology 1991; 75: A28

10. Borgeat A, Wilder-Smith OHG, Suter PM. The non-hypnotic therapeutic applications of propofol. Anesthesiology 1994; 80: 642-56

11. Herrick IA, Gelb AW, Nichols B, Kirby J. Patientcontrolled propofol sedation for elderly patients: safety and patient attitude toward control. Can. $\mathcal{F}$. Anaesth. 1996; 43(10): 1014-8

12. Pac-Soo CK, Deacock S, Lockwood G, Carr C, Whitwam JG. Patient-controlled sedation for cataract surgery using peribulbar block. Br. F. Anaesth. 1996; 77(3): 370-4 